\title{
One signal processing method of OCT based on colored noise extended Kalman filter
}

\author{
Zeng Jingru, Li Yansong, Liu Jun \\ School of Electrical and Electronic Engineering, North China Electric Power University, Beijing 102206, China.
}

\begin{abstract}
When an optical current transformer is used to measure current, its output signal is mostly nonlinear and contains colored noise; on the other hand, when the power system is in steady state operation, the frequency is not stable. This paper proposes an optical current transformer signal processing method based on colored noise extended Kalman filter which can reduce the measurement error caused by colored noise and realize frequency tracking. The following two cases are considered: 1) the dynamic noise is colored noise; 2) the observation noise is colored noise. In these two cases, the corresponding formulas of the proposed method are analyzed and derived. Simulation results showed that the extended Kalman filtering method based on colored noise can effectively filter the colored noise, and the output waveform and output error obtained by this method are improved effectively.
\end{abstract}

\section{Introduction}

As a sensing element of the primary system, the current transformers are the data source of all relay protection devices and electric energy metering devices, which requires the high output precision of current transformers [1-2].

Compared with traditional current transformers, the optical current transformers based on Faraday Effect have $\mathrm{s}$ series of advantages, such as strong anti-electromagnetic interference ability, good insulation properties and large dynamic response range[3]. However, there are still some problems in the practical application of optical current transformers.

When measuring a low current, the internal noise of the optical current transformers may be larger than that of the electro-optical signal, which results in low output precision and difficulty in separating $\mathrm{AC}$ and $\mathrm{DC}$ components. In order to solve these problems, Kalman filter is usually used to process the output signal of the optical current transformers.

The traditional Kalman filter is a linear filtering method, which requires that the state noise and observation noise of the system are all Gauss white noise [4-5].

But in the operation of the power system, most of the systems are nonlinear, and the output of the optical current transformers include some colored noises, such as $1 / \mathrm{f}$ noise, integrated noise and sinusoidal noise[6]. In addition, the frequency of the power system is not stable in actual operation, but the traditional Kalman filter method is difficult to achieve frequency tracking.

At present, many scholars have studied the use of Kalman filter based on colored noise. CS Zhao adopted the method of model compensation to modify the covariance of one-step prediction and the filter gain [7]. Considering the nonlinear problem of system, $\mathrm{T}$ Zou presented an unscented Kalman filtering method which used the observation information expansion method to modify the colored noises[8].

Based on the above problems, this paper presents a signal processing method of optical current transformers based on colored noise extended Kalman filter, and deduces the extended Kalman filtering formula of the nonlinear system in two cases of colored state noises and colored observation noises.

\section{Extended Kalman filter method}

The functional model of extended Kalman filter method is

$$
\begin{gathered}
X(k+1)=f[X(k)]+\Gamma \omega(k) \\
Y(k)=h[X(k)]+v(k)
\end{gathered}
$$

where, $X(\mathrm{k})$ is the system state vector of time $\mathrm{k} ; Y(\mathrm{k})$ is the system observation vector of time $\mathrm{k} ; \omega(\mathrm{k})$ and $v(\mathrm{k})$ are the state noise and observation noise respectively. It is assumed that $\mathrm{E}[\omega(\mathrm{k})]=0, \mathrm{E}[v(\mathrm{k})]=0, \mathrm{E}\left[\omega(\mathrm{j}) \omega^{\mathrm{T}}(\mathrm{k})\right]=Q \delta_{\mathrm{kj}}$, $\mathrm{E}\left[v(\mathrm{j}) v^{\mathrm{T}}(\mathrm{k})\right]=R \delta_{\mathrm{kj}}, \mathrm{E}\left[\omega(\mathrm{k}) v^{\mathrm{T}}(\mathrm{k})\right]=0, \mathrm{E}[\mathrm{X}(0)]=\mu_{0}$.

The recursive formulas of the extended Kalman filter under white noise are as followed.

One-step prediction:

$$
X(k+1 \mid k)=f[X(k \mid k)]
$$

The covariance of one-step prediction: 


$$
P(k+1 \mid k)=F[X(k \mid k)] P(k \mid k) F^{T}[X(k \mid k)]+\Gamma Q \Gamma^{T}
$$

Filter gain:

$$
\begin{aligned}
& K(k+1)=P(k+1 \mid k) H^{T}[X(k+1 \mid k)] \\
& \cdot\left\{H[X(k+1 \mid k)] P(k+1 \mid k) \cdot H^{T}[X(k+1 \mid k)]+R\right\}^{-1}
\end{aligned}
$$

Covariance updating:

$$
\begin{aligned}
& P(k+1 \mid k+1) \\
= & \{I-K(k+1) H[X(k+1 \mid k)]\} \cdot P(k+1 \mid k)
\end{aligned}
$$

State updating:

$$
\begin{aligned}
& X(k+1 \mid k+1)=X(k+1 \mid k) \\
& +K(k+1)\{Y(k+1)-h[X(k+1 \mid k)]\}
\end{aligned}
$$

In the formulas, $\left.F{ }^{*}\right]$ and $H\left[{ }^{*}\right]$ are the first derivative of $f\left[^{*}\right]$ and $h\left[^{*}\right]$, respectively.

\section{Extended Kalman filtering method with colored state noises}

If the covariance of state noise $\omega(\mathrm{j})$ and $\omega(\mathrm{k})$ is not zero, it means that the state noise is colored. It is assumed that $\omega(\mathrm{k})$ is related to adjacent elements $\omega(\mathrm{k}-1)$ and $\omega(\mathrm{k}+1)$, and non-adjacent elements are not related. In other words, the covariance matrix of the state noise is a block tridiagonal matrix [9-10].

In the nonlinear Kalman filtering method, the firstorder Taylor expansion of the system functional model is needed, and then the linearized model is obtained.

$$
\begin{aligned}
& X(k+1) \\
& =f[X(k \mid k)]+F[X(k \mid k)][X(k)-X(k \mid k)]+\Gamma \omega(k)
\end{aligned}
$$

$$
\begin{aligned}
& Y(k+1)=h[X(k+1 \mid k)] \\
& +H[X(k+1 \mid k)][X(k+1)-X(k+1 \mid k)]+v(k+1)
\end{aligned}
$$

According to the projective recursion formula, the state prediction value of time $\mathrm{k}+1$ based on the previous $\mathrm{k}+1$ state observation data is obtained.

$$
\begin{aligned}
& X(k+1 \mid k+1)=X(k+1 \mid k) \\
& +E\left[X(k+1) \varepsilon^{T}(k+1)\right] \cdot\left\{E\left[\varepsilon(k+1) \varepsilon^{T}(k+1)\right]\right\}^{-1} \varepsilon(k+1)(6) \\
& =X(k+1 \mid k)+K(k+1) \varepsilon(k+1)
\end{aligned}
$$

In the above formula, $K(\mathrm{k}+1)$ is the filter gain, $\varepsilon(\mathrm{k}+1)$ is the innovation sequence of time $\mathrm{k}+1$.

Next, one-step predictions of formula (4) and (5) are derived (in the subsequence calculations, $H$ is used instead of $H[\mathrm{X}(\mathrm{k}+1 \mid \mathrm{k})]$ to simplify writing).

$$
\begin{aligned}
& X(k+1 \mid k) \\
& =f[X(k \mid k)]+F[X(k \mid k)][X(k \mid k)-X(k \mid k)] \\
& =f[X(k \mid k)] \\
& Y(k+1 \mid k) \\
& =h[X(k+1 \mid k)]+H[X(k+1 \mid k)-X(k+1 \mid k)] \\
& =h[X(k+1 \mid k)]
\end{aligned}
$$

Then, the one-step prediction formula of time $\mathrm{k}+1$ is as followed.

$$
\begin{aligned}
& X(k+1 \mid k)=X(k+1)-X(k+1 \mid k) \\
& =f[X(k \mid k)]+F[X(k \mid k)][X(k)-X(k \mid k)] \\
& +\Gamma \omega(k)-f[X(k \mid k)] \\
& =F[X(k \mid k)][X(k)-X(k \mid k)]+\Gamma \omega(k)
\end{aligned}
$$

Thus, the covariance of one-step prediction is obtained. Due to the colored state noise, $\omega(\mathrm{k})$ is related to adjacent elements $\omega(\mathrm{k}-1)$ and $\omega(\mathrm{k}+1)$. It is known from the formula (1) that $X(k \mid k)$ is related to $(\mathrm{k}-1)$. As a result, $X(k \mid k)$ is related to $(\mathrm{k})$.

$$
\begin{aligned}
& P(k+1 \mid k)=E\left[X(k+1 \mid k) X^{T}(k+1 \mid k)\right] \\
& =F[X(k \mid k)] P(k \mid k) F^{T}[X(k \mid k)]+\Gamma Q \Gamma^{T} \\
& +\mathrm{F}[X(k \mid k)] P_{X(k \mid k), \omega(k)} \Gamma^{T}+\Gamma P_{\omega(k), X(k \mid k)} F^{T}[X(k \mid k)]
\end{aligned}
$$

In this formula, $X(k \mid k)$ is the error between state estimation value $X(k \mid k)$ and theoretical value. Therefore, $X(k \mid k)$ should have the same error property as $X(k \mid k)$. So this formula can be rewritten as

$$
\begin{aligned}
& P(k+1 \mid k)=F[X(k \mid k)] P(k \mid k) F^{T}[X(k \mid k)]+\Gamma Q \Gamma^{T} \\
& +\mathrm{F}[X(k \mid k)] P_{X(k \mid k), \omega(k)} \Gamma^{T}+\Gamma P_{\omega(k), X(k \mid k)} F^{T}[X(k \mid k)]
\end{aligned}
$$

X $(k \mid k)$ is derived based on the formula (6).

$$
\begin{aligned}
& X(k \mid k)=X(k)-X(k \mid k) \\
& =K(k) v(k)+\{I-K(k) H[X(k \mid k-1)]\} \\
& .\{F[X(k-1 \mid k-1)][X(k-1)-X(k-1)]+\Gamma \omega(k-1)\}
\end{aligned}
$$

Thus, the cross-covariance about state noise can be obtained.

$$
\begin{aligned}
& P_{X(k \mid k), \omega(k)}=E\left[X(k \mid k) \omega^{T}(k)\right] \\
& =\{I-K(k) H[X(k \mid k-1)]\} \Gamma P_{\omega(k-1), \omega(k)} \\
& P_{\omega(k), X(k \mid k)}=P_{X(k \mid k), \omega(k)}^{T}
\end{aligned}
$$

And the innovation is as followed. 


$$
\begin{aligned}
& \varepsilon(k+1)=Y(k+1)-Y(k+1 \mid k) \\
& =H X(k+1 \mid k)+v(k+1)
\end{aligned}
$$

Then,

$$
\begin{gathered}
E\left[\varepsilon(k+1) \varepsilon^{T}(k+1)\right]=H P(k+1 \mid k) H^{T}+R \\
E\left[X(k+1) \varepsilon^{T}(k+1)\right]=P(k+1 \mid k) H^{T}
\end{gathered}
$$

So, we can get the filter gain.

$$
\begin{aligned}
& K(k+1) \\
& =E\left[X(k+1) \varepsilon^{T}(k+1)\right] \cdot\left\{E\left[\varepsilon(k+1) \varepsilon^{T}(k+1)\right]\right\}^{-1} \\
& =P(k+1 \mid k) H^{T}\left[H P(k+1 \mid k) H^{T}+R\right]^{-1}
\end{aligned}
$$

The error of the state estimation value of time $k+1$ can be obtained.

$$
\begin{aligned}
& X(k+1 \mid k+1)=X(k+1)-X(k+1 \mid k+1) \\
& =X(k+1)-[X(k+1 \mid k)+K(k+1) \varepsilon(k+1)] \\
& =\{I-K(k+1) H\} X(k+1 \mid k)-K(k+1) v(k+1)
\end{aligned}
$$

(16)

The covariance updating formula is derived and simplified from the above formula.

$$
\begin{aligned}
& P(k+1 \mid k+1)=E\left[X(k+1 \mid k+1) X^{T}(k+1 \mid k+1)\right] \\
& =[I-K(k+1) H] P(k+1 \mid k)
\end{aligned}
$$

In this way, the complete recursive formulas of the extended Kalman filter is obtained in the condition of the colored state noise. It can be seen that the recursive formulas is consistent with the extended Kalman filter in the condition of the white noise in the form. When the cross-covariance term is zero, that is, the state noise is white noise, the formula is restored to the extended Kalman filter under white noise.

\section{Extended Kalman filtering method with colored observation noises}

If the covariance of observation noise $v(\mathrm{j})$ and $v(\mathrm{k})$ is not zero, it means that the state noise is colored. It is assumed that $v(\mathrm{k})$ is related to adjacent elements $v(\mathrm{k}-1)$ and $v(\mathrm{k}+1)$, and non-adjacent elements are not related. In other words, the covariance matrix of the observation noise is a block tridiagonal matrix.

The covariance of one-step prediction is obtained by formula (10).

$$
P(k+1 \mid k)=F[X(k \mid k)] P(k \mid k) F^{T}[X(k \mid k)]+\Gamma Q \Gamma^{T}
$$

Due to the colored observation noise, $v(\mathrm{k}+1)$ is related to adjacent elements $v(\mathrm{k})$ and $v(\mathrm{k}+2)$. The one-step prediction is estimated by the previous $\mathrm{k}$ state observation data, which is means that $X(k+1 \mid k)$ is related to (k). As a result, $X(k+1 \mid k)$ is related to $(\mathrm{k}+1)$. $X(k+1 \mid k)$ is the error between one-step prediction value $X(k+1 \mid k)$ and theoretical value. Therefore, $X(k+1 \mid k)$ should have the same error property as $X(k+1 \mid k)$.

$$
\begin{aligned}
& E\left[\varepsilon(k+1) \varepsilon^{T}(k+1)\right]=H P(k+1 \mid k) H^{T}+R \\
& +H P_{X(k+1 \mid k), v(k+1)}+P_{v(k+1), X(k+1 \mid k)} H^{T} \\
& E\left[X(k+1) \varepsilon^{T}(k+1)\right]=P(k+1 \mid k) H^{T}+P_{X(k+1 \mid k), v(k+1)}
\end{aligned}
$$

(20)

Then, we can get the filter gain.

$$
\begin{aligned}
& K(k+1)=\left[P(k+1 \mid k) H^{T}+P_{X(k+1 \mid k), v(k+1)}\right] \\
& \cdot\left[H P(k+1 \mid k) H^{T}+R\right. \\
& \left.+H P_{X(k+1 \mid k), v(k+1)}+P_{v(k+1), X(k+1 \mid k)} H^{T}\right]^{-1}
\end{aligned}
$$

To obtain the cross-covariance about $\(k+1 \mid k)$ and $(\mathrm{k}+1), X(k+1 \mid k)$ should be modified as followed first.

$$
\begin{aligned}
& X(k+1 \mid k)=X(k+1)-X(k+1 \mid k) \\
& =X(k+1)-f[X(k \mid k)]
\end{aligned}
$$

And,

$$
\begin{aligned}
& f[X(k \mid k)] \\
& =f\{X(k \mid k-1)+K(k)[H X(k \mid k-1)+v(k)]\}
\end{aligned}
$$

To simplify the calculation, let

$$
g[v(k)]=f\{X(k \mid k-1)+K(k)[H X(k \mid k-1)+v(k)]\}
$$

In order to obtain the relationship between $X(k+1 \mid k)$ and $(\mathrm{k})$, a first-order Taylor expansion is performed on $g[v(\mathrm{k})]$ at $v(\mathrm{k})=0$, thus

$$
\begin{aligned}
& X(k+1 \mid k)=X(k+1)-g[v(k)] \\
& =X(k+1)-g[\bar{v}(k)]-g^{\prime}[\bar{v}(k)][v(k)-\bar{v}(k)] \\
& =X(k+1)-g(0)-g^{\prime}(0) v(k)
\end{aligned}
$$

Then,

$$
\begin{gathered}
P_{X(k+1 \mid k), v(k+1)}=-g^{\prime}(0) P_{v(k), v(k+1)} \\
P_{v(k+1), X(k+1 \mid k)}=P_{X(k+1 \mid k), v(k+1)}^{T}
\end{gathered}
$$

According to the formula (16), the covariance updating formula is derived.

$$
\begin{aligned}
& P(k+1 \mid k+1)=E\left[X(k+1 \mid k+1) X^{T}(k+1 \mid k+1)\right] \\
& =[I-K(k+1) H] P(k+1 \mid k)[I-K(k+1) H]^{T} \\
& +K(k+1) R K^{T}(k+1) \\
& -[I-K(k+1) H] P_{X(k+1 \mid k), v(k+1)} K^{T}(k+1) \\
& -K(k+1) P_{v(k+1), X(k+1 \mid k)}[I-K(k+1) H]^{T} \\
& =[I-K(k+1) H] P(k+1 \mid k)-K(k+1) P_{v(k+1), X(k+1 \mid k)}
\end{aligned}
$$

In conclusion, the complete recursive formulas of the extended Kalman filter is obtained in the condition of the 
colored observation noise. It also can be seen that the recursive formulas is consistent with the extended Kalman filter in the condition of the white noise in the form. When the cross-covariance term is zero, that is, the observation noise is white noise, the formula is restored to the extended Kalman filter under white noise.

\section{Experimental results and comparison}

Considering the frequency offset, the following output model is obtained.

$$
y=A \sin (2 \pi k f T+2 \pi k \Delta f T+\varphi)+C
$$

In the above formula, $f$ is the fundamental frequency of the power system, $\Delta f$ is the offset of frequency based on the fundamental frequency $f$, and $\mathrm{T}$ is the sampling interval.

According to the formula for summation of angles,

$$
\begin{aligned}
& y=A \sin (2 \pi k f T+2 \pi k \Delta f T) \cos \varphi \\
& +A \cos (2 \pi k f T+2 \pi k \Delta f T) \sin \varphi+C
\end{aligned}
$$

It's selected that $x_{1}=\Delta f, x_{2}=\mathrm{A} \cos \varphi, x_{3}=\mathrm{A} \sin \varphi$, the noise driving matrix $\Gamma$ is $I$, the state driving matrix is $I$, Then the functional model is

$$
\begin{gathered}
X(k+1)=X(k)+\omega(k) \\
Y(k+1)=h[X(k+1)]+v(k+1)
\end{gathered}
$$

where, $\mathrm{E}[\omega(\mathrm{k})]=0, \mathrm{E}[v(\mathrm{k})]=0, \mathrm{E}\left[\omega(\mathrm{j}) \omega^{\mathrm{T}}(\mathrm{k})\right]=Q \delta_{\mathrm{kj}}, \mathrm{E}[v(\mathrm{j})$ $\left.v^{\mathrm{T}}(\mathrm{k})\right]=R \delta_{\mathrm{kj}}, \mathrm{E}\left[\omega(\mathrm{k}) v^{\mathrm{T}}(\mathrm{k})\right]=0, \mathrm{E}[\mathrm{X}(0)]=\mu_{0}$.

Based on the above formulas, experimental verification and comparison of results are made. When the white noise is superimposed, the traditional extended Kalman filtering method, the extended Kalman filtering method under colored state noise and colored observation noise are used respectively. The output waveforms as shown in Figure 1. It can be seen that the three filter results are basically the same, indicating the extended Kalman filter under colored noise is also suitable when the noise is white noise.

When the colored state noise is superimposed, the traditional extended Kalman filtering method, the extended Kalman filtering method under colored state noise and colored observation noise are used respectively. The output waveforms of the three methods as shown in Figure 2. It can be seen that the waves of extended Kalman filtering method under the colored state noise are smoother and less error, and the filtering effect is better than that of the traditional extended Kalman filtering method.

When the colored observation noise is superimposed, the traditional extended Kalman filtering method, the extended Kalman filtering method under colored state noise and colored observation noise are used respectively. The output waveforms of the three methods as shown in Figure 3. It can be seen that when the extended Kalman filtering method under colored observation noise is applied, the output waveform fluctuates less and is more stable, which can confirm the effectiveness of the method.

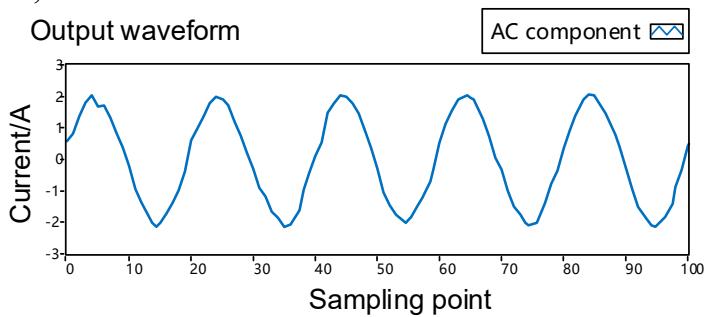

(a) The AC waveform of the EKF under white noise when white noise is superimposed

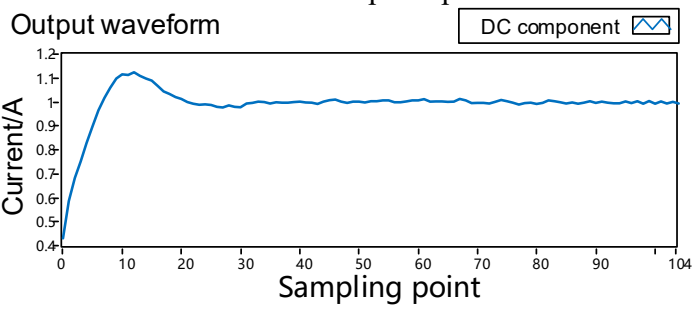

(b) The DC waveform of the EKF under white noise when white noise is superimposed

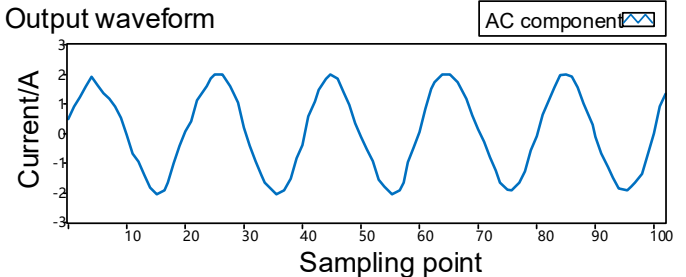

(c) The AC waveform of the EKF under colored state noise when white noise is superimposed

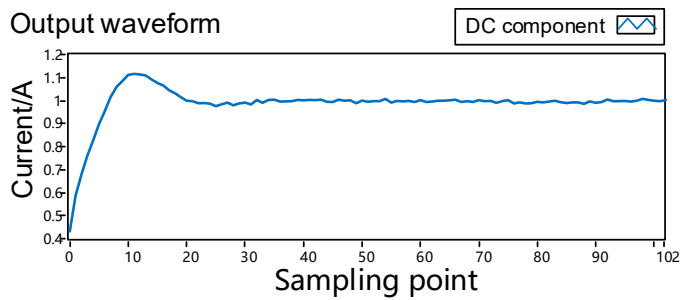

(d) The DC waveform of the EKF under colored state noise when white noise is superimposed

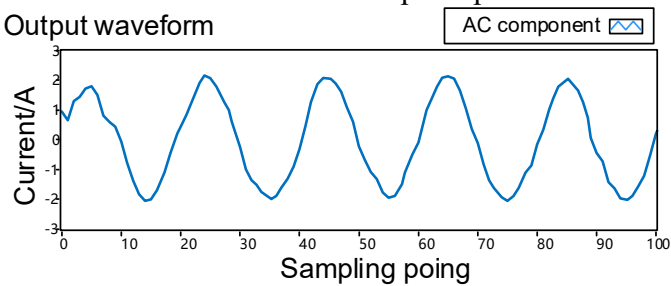

(e) The AC waveform of the EKF under colored observation noise when white noise is superimposed

\begin{tabular}{ll} 
Output waveform & DC component $\square$ \\
\hline
\end{tabular}

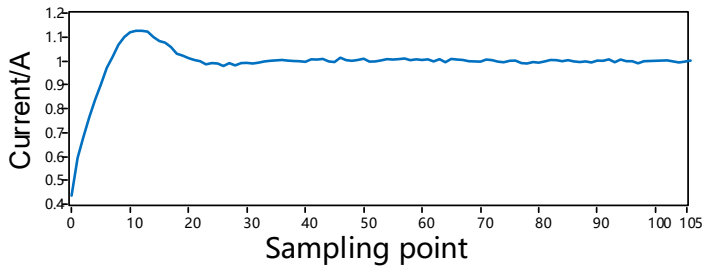

(f) The DC waveform of the EKF under colored observation noise when white noise is superimposed

Figure 1. Comparison of output results of different filtering methods when white noise is superimposed 


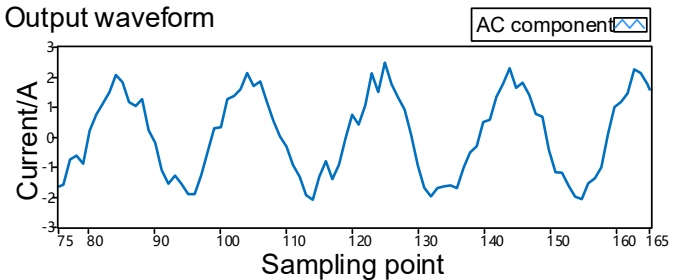

(a) The AC waveform of the EKF under white noise when colored state noise is superimposed

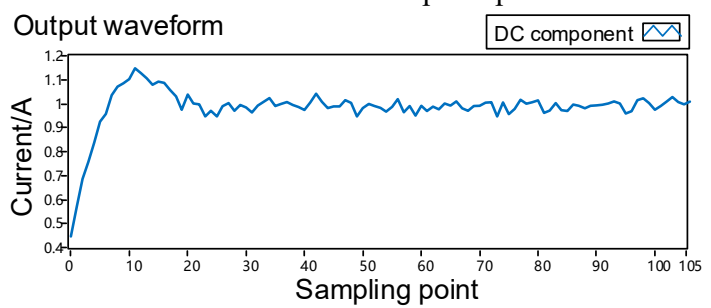

(b) The DC waveform of the EKF under white noise when colored state noise is superimposed

Output waveform

AC component $\mathbb{Q}$

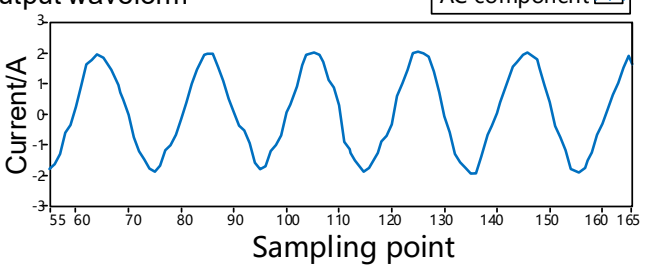

(c) The AC waveform of the EKF under colored state noise when colored state noise is superimposed

Output waveform DC component

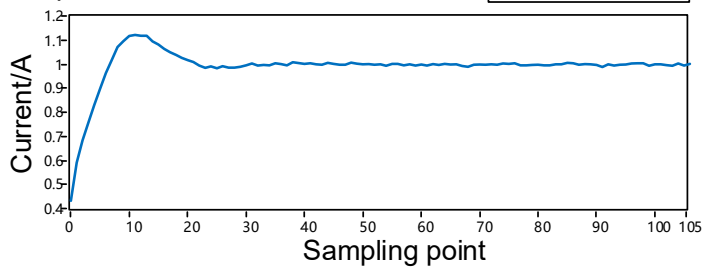

(d) The DC waveform of the EKF under colored state noise when colored state noise is superimposed

Figure 2. Comparison of output results of different filtering methods when colored dynamic noise is superimposed Output waveform

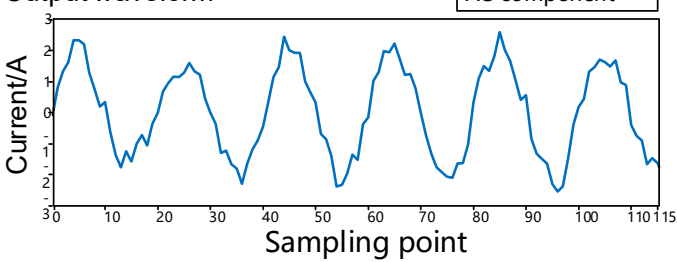

(a) The AC waveform of the EKF under white noise when colored observation noise is superimposed

Output waveform

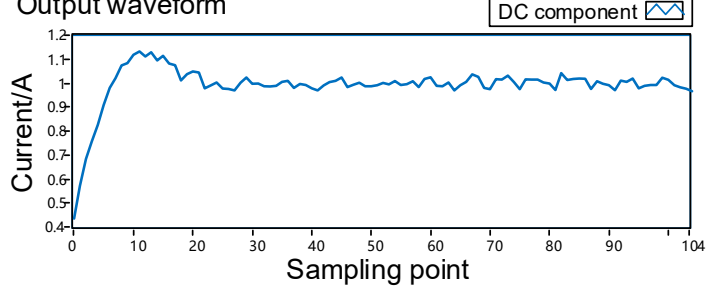

(b) The DC waveform of the EKF under white noise when colored observation noise is superimposed

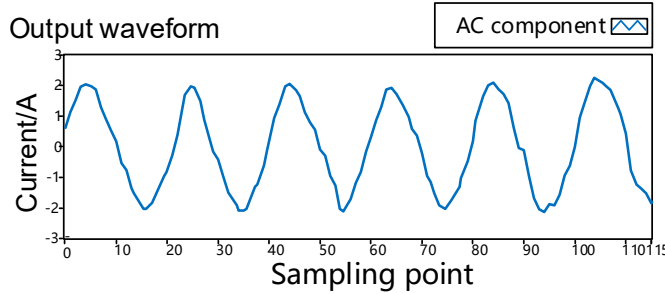

(c) The AC waveform of the EKF under colored observation noise when colored observation noise is superimposed Output waveform DC component $₫$

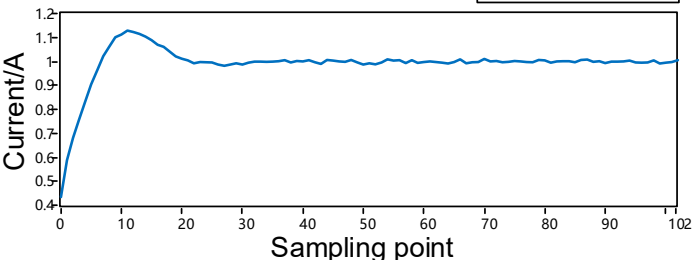

(d) The DC waveform of the EKF under colored observation noise when colored observation noise is superimposed

Figure 3. Comparison of output results of different filtering methods when colored observation noise is superimposed

\section{Conclusion}

When the power system is in normal operation, the output signals of the optical current transformer are mostly nonlinear, and there are problems such as frequency fluctuation and noise color. A large error may be caused when the output signal is processed by the traditional Kalman filtering method. In this paper, considering the nonlinearity, frequency fluctuation, colored noise of the system, a method of extended Kalman filtering in the condition of colored noise applied to optical current transformer signal processing is proposed.

In this paper, the output of different Kalman filtering methods under various noise conditions is experimentally compared. It is proved that the extended Kalman filtering method in the condition of colored noise can be effectively applied to nonlinear systems with colored noise. In the condition of colored noise, the filtering effect of the optical current transformer output signal is better than the traditional Kalman filtering method. It can separate the frequency components needed in the output signal with high precision, make the output waveform more smooth, reduce the filtering error, and improve the accuracy of filtering.

\section{Reference}

1. Z Zhang. Huazhong University of Science and Technology, (2013)

2. ZZ Guo. PSPC, 15, 1-5, (2008)

3. YH Yan. North China Electric Power University, (2016)

4. ZF Zhang, J Liu, YS Li, BJ Li. EMI, 54, 83-88+116, (2017)

5. YS Li, X Li, J OuYang, YH Yan. EMI, 53, 16-21, (2016)

6. YS Li, J OuYang, J Liu, LX Cao. AEPS, 39, 126129+137, (2015) 
7. CS Zhao, BZ Tao. GISWU, 2, 180-182+207, (2008)

8. T Zou, CS Zhao, ZX Ding. BSM, 6, 24-27, (2015)

9. X Xiong, MH Guo, WJ Li, J Zhao, JH Cui. JCIT, 25, 33-36, (2017)

10. F Chen, XY Wu, WY Liu, F Liu. JXUPT, 19, 56-59, (2014) 